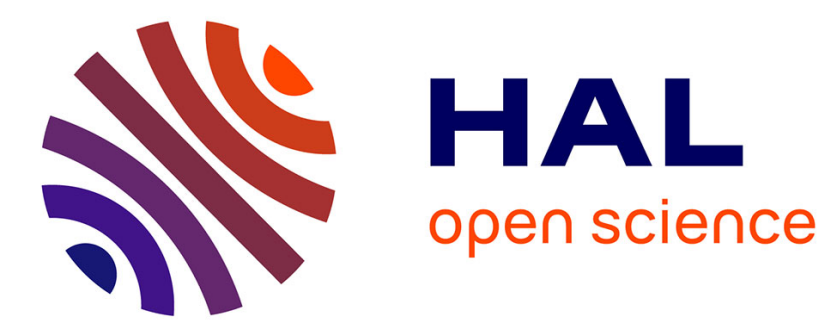

\title{
Fourier-based geometric shape prior for snakes
}

Mohamed-Ali Chermi, Stéphane Derrode, Faouzi Ghorbel

\section{To cite this version:}

Mohamed-Ali Chermi, Stéphane Derrode, Faouzi Ghorbel. Fourier-based geometric shape prior for snakes. Pattern Recognition Letters, 2008, 29 (7), pp.897-904. 10.1016/j.patrec.2008.01.011 . hal00325106

\section{HAL Id: hal-00325106 https://hal.science/hal-00325106}

Submitted on 26 Sep 2008

HAL is a multi-disciplinary open access archive for the deposit and dissemination of scientific research documents, whether they are published or not. The documents may come from teaching and research institutions in France or abroad, or from public or private research centers.
L'archive ouverte pluridisciplinaire HAL, est destinée au dépôt et à la diffusion de documents scientifiques de niveau recherche, publiés ou non, émanant des établissements d'enseignement et de recherche français ou étrangers, des laboratoires publics ou privés. 


\title{
Fourier-based geometric shape prior for snakes
}

\author{
M.-A. Charmi ${ }^{a}$, S. Derrode ${ }^{\text {b,* }}$ and F. Ghorbel ${ }^{\text {a }}$ \\ ${ }^{a}$ GRIFT Group, CRISTAL Laboratory, \\ École Nationale des Sciences de l'Informatique, \\ Campus Universitaire de la Manouba, \\ 2010 Manouba, Tunisia. \\ charmi.ma@free.fr, faouzi.ghorbel@ensi.rnu.tn \\ b GSM, Institut Fresnel (CNRS UMR 6133), \& École Centrale Marseille, \\ Technopôle de Château-Gombert, 38, rue Frédéric Joliot Curie, \\ 13451 Marseille Cedex 20, France. \\ stephane.derrode@ec-marseille.fr
}

\begin{abstract}
A novel method of snakes with shape prior is presented in this paper. We propose to add a new force which makes the curve evolve to particular shape corresponding to a template to overcome some well-known problems of snakes. The template is an instance or a sketch of the researched contour without knowing its exact geometric pose in the image. The prior information is introduced through a set of complete and locally stable invariants to Euclidean transformations (translation, rotation and scale factor) computed using Fourier Transform on contours. The method is evaluated with the segmentation of myocardial scintigraphy slices and the tracking of an object in a video sequence.
\end{abstract}

Key words: Snakes, shape prior, Fourier transform, invariant, completeness, object tracking.

\section{Introduction}

Since Kass et al. seminal paper in 1987 [17], many researchers investigated in active contour methods. Several variants have been presented. One can classify

* Corresponding author. 
them in explicit models, i.e. snakes [17] and implicit ones, i.e. level sets [2]. Active contours can treat contours as a whole contrary to the classic contour detectors (filters such as Sobel, Chen or Canny-Deriche) in which the output is a set of isolated points characterized by strong gradient.

During the last two decades, several improvements of the snakes original model were proposed. Many approaches have been introduced to minimize the snakes energy such as variational calculus [17], greedy algorithm [26], dynamic programming [1] and finite elements method [6]. It is well known that snakes suffer from two major problems: their sensitiveness to the initial position of the curve and their inadequacy for concave boundaries. To cope with these problems, some solutions have been proposed in literature. Cohen [5] adds new forces called the balloon forces which move the curve in the absence of external energy. Balloon forces are normal to the curve and their weight is not too important to allow the snakes to stop at the right edges. A prior knowledge of the position of the initial curve is needed to know if the balloon inflates or deflates. This method enhances sensitiveness to the initial position of the curve. Gradient Vector Flow (GVF) is an external energy computed using the general diffusion equations. The GVF, to some extent, has a large capture range and presents forces that enable the snakes to evolve in concave boundaries. Some other models have been presented such as Dual Snakes [15] and Multiresolution snakes [19]. The fields of applications of these methods include motion tracking [4], stereovision $[4,12,18]$, medical imagery [5, 6] and remote sensing [24].

Active contours, like most methods of segmentation, use essentially gray levels of pixels that are low-level primitives. Sometimes, results are not satisfactory, especially in presence of disruptive elements such as noise and occlusions. This motivates the need to add prior. Snakes were among the first methods allowing prior information embedding through the internal energy in the initial model of Kass [17]. This internal energy adds constraints of elasticity and of rigidity of the curve.

During the last decade, several investigations proposed to integrate prior information into active contour models. First, Staib and Duncan [25] suggest a new description of the contours by the elliptic Fourier descriptors and associate each object to a shape class using Gaussian probability distributions. Diffusion Snakes [7] are a modification of the Munford-Shah functional that allows explicit expression of the curve. Thus, statistical shape prior using a set of training data can be added to the functional of energy. The minimisation is done in a variational framework. A Bayesian framework is used in [29] to compute prior information obtained from a prototype. Transformations between the prototype and the researched edges are estimated using the least square fitting. Shape descriptors have been recently used to add prior information to region-based active contours. Foulonneaux et al. [10] use Legendre moments to 
make the contour evolve to a shape of reference. It introduces a quadratic distance between the contour and the object of reference. This energy is invariant to translation and scale factor. Rotation can be taken into consideration, yet the computation may still more complex. Rochery et al. [24] presented a new generation of active contours called High Order Active Contours. It allows the integration of geometric information expressed in interaction between different points of the contour, contrary to linear classical energies. This method has been applied in the detection of linear primitives such as roads and rivers in remote sensing data.

Prior knowledge enhances the active contour results. Indeed, it increases the methods robustness to noise, clutter and occlusions. In addition, it increases the active contour convergence. However, the prior embedding in these models can increase their complexity, which can affect their applicability in real time applications such as motion tracking. Prior information may not be available in some cases.

This paper is organized as follows: in section 2, we recall some principles of snake models. An overview of shape description methods using Fourier is presented in section 3. The main contribution of this work including a novel method of snakes with shape prior is detailed in section 4 . The fourth section is devoted to experimental results on synthetic images. In section 5 we present the application of the method to the segmentation of myocardial scintigraphy images and to object tracking in video sequences. Finally, we conclude and highlight some perspectives for further investigation.

\section{Overview of snakes methods}

Snakes are methods for edge detection by energy minimization of a planar curve. The curve is given by its parametric representation $v(s, t)=(x(s, t), y(s, t))$ where $t$ denotes the time and $s$ the normalized arclength. The energy of the snake is

$$
E_{\text {snakes }}^{*}=\int_{0}^{1}\left(E_{\text {int }}+E_{\text {ext }}\right) d s .
$$

$E_{\text {int }}$ is the internal energy

$$
E_{\text {int }}=\int_{0}^{1} \alpha\left|v^{\prime}(s)\right|^{2}+\beta\left|v^{\prime \prime}(s)\right|^{2} d s,
$$

where $v^{\prime}(s)$ and $v^{\prime \prime}(s)$ are the first and second derivatives of $v$ according to $s$. The first term of the internal energy is called the elasticity term. It prevents the apparition of isolated points in the curve. The second one is the bending term which prevents the formation of corners and sharp angles in the contour. 
These two terms correspond to the internal energy and make the snakes look like a thin plate. $\alpha$ and $\beta$ are two weight parameters.

$E_{\text {ext }}$ is the image or external energy which attracts the snake to features of interest (lines or edges). In general, $E_{\text {ext }}$ is the smoothed image gradient:

$$
E_{e x t}=-\left|\nabla\left(G_{\sigma} * I\right)\right|,
$$

where $I$ is a gray level image, $G_{\sigma}$ a bi-dimensional Gaussian filter with standard deviation $\sigma$ and $\nabla$ the gradient operator.

\section{Overview of Fourier-based shape description methods}

This section recalls some basic facts about shape description of closed planar curves under the action of Euclidean transformations, see [14] for a detailed presentation. In this case, shapes can be parameterized according to the normalized arclength:

$$
\begin{aligned}
f[0,1] & \rightarrow \mathbb{C} \\
l & \mapsto f(l)=\frac{1}{L} \int_{0}^{l}\left|f^{\prime}(u)\right| d u,
\end{aligned}
$$

where $L$ denotes the curve length. We say that two objects $O_{1}$ and $O_{2}$ have the same shape according to Euclidean transformations, if, for all parameterizations $f_{1}$ and $f_{2}$ of $O_{1}$ and $O_{2}$, we can write:

$$
f_{2}(l)=e^{j \theta} f_{1}\left(l+l_{0}\right),
$$

where $\theta$ denotes the orientation difference and $l_{0}$ the starting description points difference. Scale factor between the two curves is not considered since arclength parametrization are normalized, i.e. curves have an equal length of 1 . Also we do not consider any translation between the two objects since curves are described according to their center of mass.

Since parameterizations are periodic, Fourier series $\left\{C_{k}(.)\right\}_{k \in J}, J=\left[-\frac{N}{2} ; \frac{N}{2}-\right.$ 1] can be computed, and we get the following relation between $O_{1}$ and $O_{2}$ in the Fourier domain:

$$
\forall k \in J, \quad C_{k}\left(f_{2}\right)=e^{j\left(\theta+2 \pi k l_{0}\right)} C_{k}\left(f_{1}\right) .
$$

The search for invariant Fourier descriptors comes from the nice relation above, also called shift theorem. We recall that a set of scalars $\left\{I_{k}\right\}_{k \in J}$ is invariant 
with respect to Euclidean transformations if and only if, for two objects $O_{1}$ and $O_{2}$ with the same shape, we get $I_{k}\left(f_{1}\right)=I_{k}\left(f_{2}\right)$ for all $k$ in $J$. Initially, the first set of invariants was constructed by taking the modulus of Fourier descriptors

$$
\forall k \in J, \quad I_{k}(f)=\left|C_{k}(f)\right|,
$$

and used to discriminate between simple-shaped objects $[21,28]$.

Nevertheless, this set is not complete in the sense defined in [8]. A set of descriptors is said to be complete if the following property is verified: two objects have the same shape if and only if they have the same set of invariants. Completion allows the reconstruction of shapes from their invariants, up to an Euclidean transformation. In fact, the set of descriptors in equation (7) is no complete since we can find objects with different shapes but with the same magnitude for their Fourier coefficients (the phase information is lost). To overcome this problem, T.R. Crimmins [8] proposed the following complete set:

$$
\begin{aligned}
& I_{k_{0}}(f)=\left|C_{k_{0}}(f)\right|, \quad \text { for } k_{0} \text { such that } C_{k_{0}}(f) \neq 0 \\
& I_{k_{1}}(f)=\left|C_{k_{1}}(f)\right|, \quad \text { for } k_{1} \neq k_{0} \text { such that } C_{k_{1}}(f) \neq 0 \\
& I_{k}(f)=C_{k}(f)^{k_{0}-k_{1}} C_{k_{0}}(f)^{k-k_{1}} C_{k_{1}}(f)^{k_{0}-k}, \quad \forall k \neq k_{0}, k_{1} .
\end{aligned}
$$

However, this set is not stable, i.e. a slight modification of invariants may induce a noticeable shape distortion. A complete and stable set of invariant Fourier descriptors has then been presented in [13]:

$$
\begin{aligned}
& I_{k_{0}}(f)=\left|C_{k_{0}}(f)\right|, \quad \text { for } k_{0} \text { such that } C_{k_{0}}(f) \neq 0 \\
& I_{k_{1}}(f)=\left|C_{k_{1}}(f)\right|, \quad \text { for } k_{1} \neq k_{0} \text { such that } C_{k_{1}}(f) \neq 0 \\
& I_{k}(f)=\frac{C_{k}(f)^{k_{0}-k_{1}} C_{k_{0}}(f)^{k-k_{1}} C_{k_{1}}(f)^{k_{0}-k}}{I_{k_{0}}(f)^{k-k_{1}-p} I_{k_{1}}(f)^{k_{0}-k-q}}, \quad \forall k \neq k_{0}, k_{1},
\end{aligned}
$$

with $p, q>0$.

All these properties have been extended to the action of affine transformations on closed planar curves $[3,14]$.

\section{Fourier-based shape prior for snakes}

We propose to use a set of Fourier-based shape invariants to constrain the snakes evolution to a particular shape called template which represents the prior information. We exploit some specific properties of the invariants such as completeness. 


\subsection{Presentation of the shape-invariant family}

Let $v$ be a discrete parametrization of a closed curve with $N$ points: $v(n)=$ $x(n)+j y(n) ; n=1, \ldots, N$; where $x(n)$ and $y(n)$ are given according to the barycenter of $v$. Let $C_{k}(v)$ denote the Discrete Fourier Transform (DFT) of $v$ :

$$
C_{k}(v)=\frac{1}{N} \sum_{n=0}^{N-1} v(n) e^{-j \frac{2 \pi n k}{N}},
$$

for $k=-\frac{N}{2}, \ldots, \frac{N}{2}-1$. The set of complex coefficients

$$
\begin{aligned}
I_{k_{0}}(v) & =\left|C_{k_{0}}(v)\right| \\
I_{k}(v) & =\frac{C_{k}(v)}{C_{k_{0}}(v)}, k=-\frac{N}{2}, \ldots, \frac{N}{2},
\end{aligned}
$$

forms a complete [8] and locally stable family of shape descriptors which are invariant to translation, rotation and scale factor, but not to the initial description point. The shape can be retrieved using the inverse Fourier transform (iDFT).

From a numerical point of view, no general rule can be given to choose an optimal number of points $N$ for the parametrization. This number must not be too big for computational burden reasons and not too small to avoid a too smooth approximation of shapes. Generally, as we use the FFT algorithm for reducing the computing time for both DFT and iDFT computations, we take for $N$ the power of 2 just less than the number of pixels of the original contour.

\subsection{Invariants embedding in snakes}

To introduce shape prior information, we add a new force that guides the active contour in the image to a given template $v_{\text {ref }}$, independently of its pose, orientation and size. A two-step strategy is adopted. At each step $t$ of the algorithm:

(1) First, we compute a linear mixture of the snake invariants at time $t$ and the template invariants according to

$$
I_{k}\left(v_{t}^{\prime}\right)=\left(1-c_{k, t}\right) I_{k}\left(v_{t}\right)+c_{k, t} I_{k}\left(v_{r e f}\right),
$$

where $c_{k, t} \in[0,1]$ is a weight function which depends on the harmonic order $k$ and time $t . c_{k, t}$ can be constant or a low-pass filter (e.g. Hamming window) to give more importance to low-order harmonics. $I_{k}\left(v_{t}^{\prime}\right)$ can be considered as the invariants set of a curve $v_{t}^{\prime}$ influenced by the classical snakes evolution $v_{t}$ 
and by the template contour $v_{r e f}$. Equation (11) is the homotopy function. $v_{t}$ must be re-sampled at each iteration in order to have the same distance between each two consecutive nodes of the snake.

(2) Second, we reconstruct $v_{t}^{\prime}$ from $I_{k}\left(v_{t}^{\prime}\right)$ using the completeness property of the invariants set,

$$
\begin{aligned}
C_{k}\left(v_{t}^{\prime}\right) & =C_{k_{0}}\left(v_{t}^{\prime}\right) I_{k}\left(v_{t}^{\prime}\right), \\
v_{t}^{\prime}(n) & =\frac{1}{N} \sum_{k=0}^{N-1} C_{k}\left(v_{t}^{\prime}\right) e^{j \frac{2 \pi n k}{N}},
\end{aligned}
$$

and computing the iDFT. Since harmonic $C_{k_{0}}$ of curve $v_{t}^{\prime}$ is unknown, we set $C_{k_{0}}\left(v_{t}^{\prime}\right)$ to $C_{k_{0}}\left(v_{t}\right)$. Hence, $v_{t}^{\prime}$ is reconstructed with the same pose than $v_{t}$. The parameter $k_{0}$ is chosen, at each iteration, so that $C_{k_{0}}$ is big compared to other harmonics to avoid division by small numbers.

We next define the prior shape forces as the difference between the snake $v_{t}$ and the reconstructed shape $v_{t}^{\prime}$ after the invariants modification: $F_{\text {prior }}=v_{t}^{\prime}-v_{t}$. The forces of the snake become $F=c_{2} F_{\text {prior }}^{t}-\nabla E_{\text {ext }}\left(v_{t}\right)$, where $c_{2}$ is a constant weight. We recall here that the two curves must have the same starting point.

To enhance numerical stability, we normalize the prior forces and $E_{\text {ext }}$ in the same way as in $[5,25]$. The new forces of snakes are then defined as follows:

$$
F=c_{2} \frac{F_{\text {prior }}^{t}}{\left|F_{\text {prior }}^{t}\right|}-c_{3} \frac{\nabla E_{\text {ext }}\left(v_{t}\right)}{\left|\nabla E_{\text {ext }}\left(v_{t}\right)\right|}
$$

where $c_{3}$ is another constant weight that regularizes the prior and image forces.

\section{$5 \quad$ Experimental results}

To assess the performance of the method, we propose a set of experimental results on syntectic and real data. Here we evaluate the behavior of the new forces. We also show how the method can solve the problem of snakes evolution in concave boundaries. We study the influence of parameters and show how the curve evolves under the prior forces only.

Figure 1 portrays the evolution of some curves only under the prior forces action. The external and the internal energies are not considered. It is clear that the curves converge to have the same shape of the given template.

The major problem of snakes is their inadequacy in concave boundaries. The results of the snakes with shape prior on the $U$ shape with different cases of 

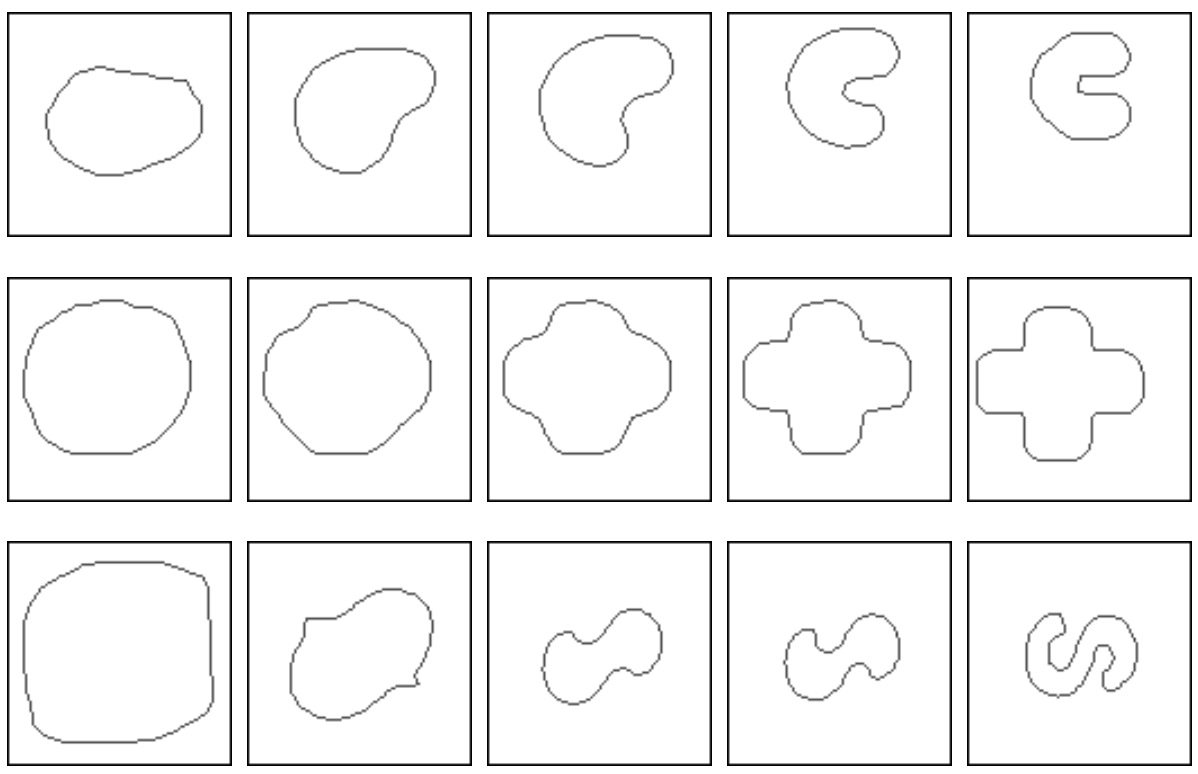

Fig. 1. Curves moved under the prior forces only.

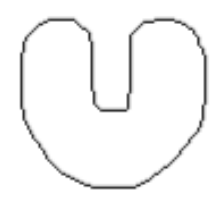

(a) template

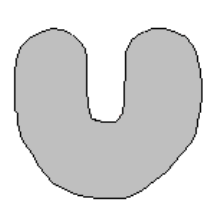

(b)

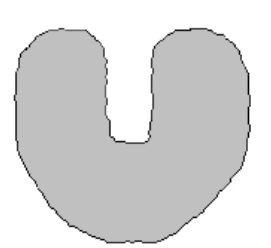

(c)

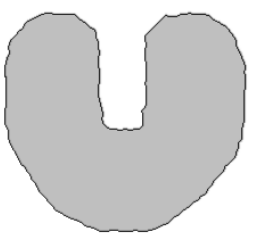

(d)

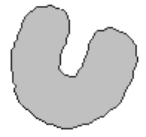

(e)

Fig. 2. Results on the U shape using (a) as template. (b) no transformations, (c) scale factor, (d) translation + scale factor, (e) rotation + scale factor.

Euclidean Transformations are presented in figure 2. These examples show the snakes ability to solve the problem. Indeed, the classical snakes energies does not attract the curve inside the concavity. The addition of the prior knowledge allows the snakes to evolve in concave boundaries. In fact, the existence and localisation of the concavities are carried by the invariants.

The GVF method is the only model that copes with the problem of evolving into concave boundaries. But, the GVF snakes are not able to evolve to highly concave boundaries. In such case, the presented model gives better results. In fact, in figure 3, we compare the results of our method to the GVF 


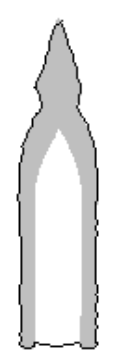

(a)

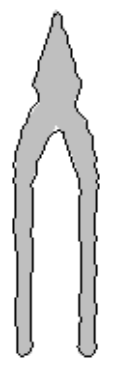

(b)

Fig. 3. The ability of snake with shape prior to evolve in highly concave boundaries. (a) GVF snakes [27], (b) our method.

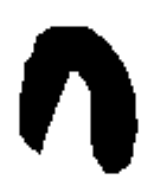

(a)

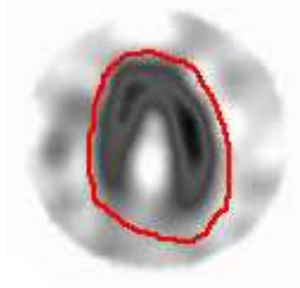

(b)

Fig. 4. Initial conditions of myocardial scintigraphy images segmentation using snakes with shape prior: (a) template used for images, (b) initial curve.

snakes on the object pliers which is characterized by their deep concavities. We successfully segment the pliers whereas the GVF snakes fail.

\section{Some Applications}

In what follows, we summarize the results of the method applied to myocardial scintigraphy images and to object tracking.

\subsection{Myocardial scintigraphy images}

First, we apply our method to the segmentation of myocardial scintigraphy images on effort (after activity). The reference curve has been obtained by running a classical snakes algorithm. The initial curve was placed inside the concavity. Some points that seemed wrong were removed or fixed by a human operator. This approximate template shown in figure 4(a) was used for all the treated images presented in figure 5. This experiment can be seen as a validation of the method robustness to approximate prior (sketch). In fact, image forces are usually more important than shape forces. The values of the 


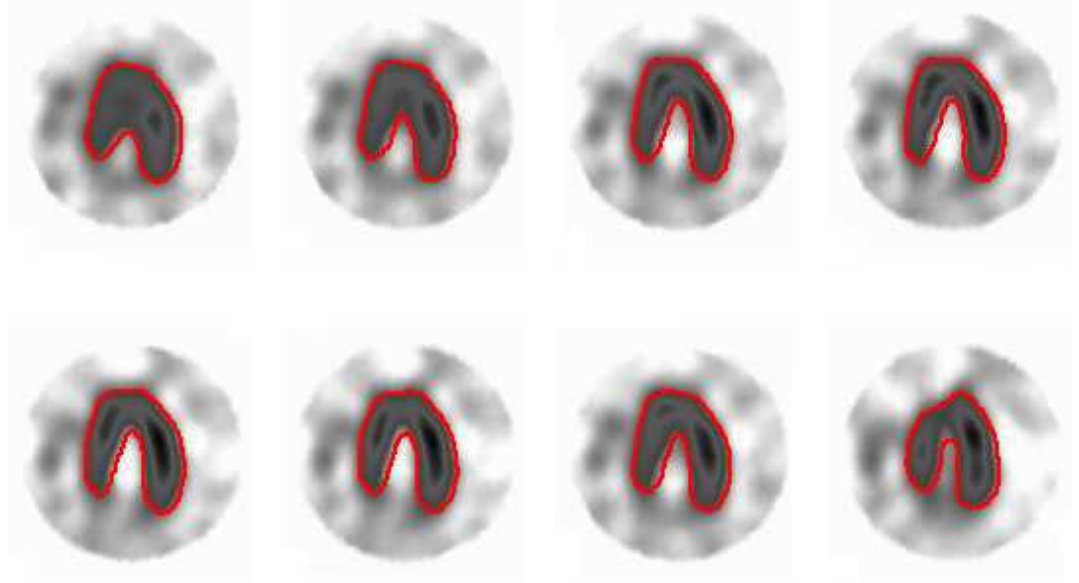

Fig. 5. Results of the snakes with shape prior on myocardial scintigraphy images. introduced parameters are: $c_{1}=0.1, c_{2}=0.25$ and $c_{3}=0.3$. The contour is re-sampled into 100 points. An example of initial curve is given by figure 4(b). The obtained results are shown in figure 5. They appear visually satisfactory. The snakes evolve successfully in the concave zones.

Our results are satisfactory compared to those obtained by the watershed segmentation method corrected by ground truth from experts. They can be used for ischemia diagnosis by comparing the myocardium in rest and effort.

\subsection{Object tracking}

Due to their low complexity, snakes have been widely used in object tracking. In [23], snakes are used to follow cells in microscopic images. Hao et al. present a predictive snake [16] for general object tracking. The inertia of the tracked object is predicted using block-wise motion estimation and a smoothing process. Kalman snakes [22] use gradient-based measurements and optical flow along contours for tracking non rigid objects. The method is more robust to occlusion and clutter.

We then considered the tracking of an isolated object using the snakes with shape prior. The use of the prior knowledge constrains the snakes evolution to the tracked shape. The adopted strategy consists in using the contour obtained at frame $t_{i-1}$ as a template for the snakes at frame $t_{i}$. We assume that the shape deformations between two consecutive treated frames are not important.

In our experiments, we used three sequences. The first two ones represent a moving cup and a moving hand, and the third one a fixed mouse acquired from a moving camera. Figure 6 shows the tracking result of some frames of the "cup sequence" with a quite uniform background and high contrast. 


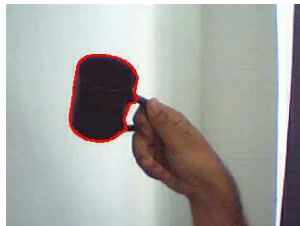

(a) frame 35

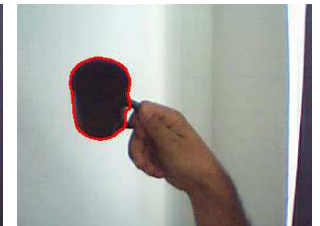

(b) frame 68

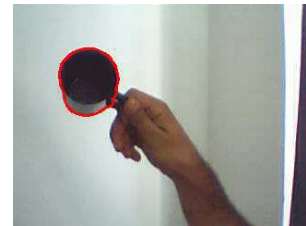

(c) frame 139

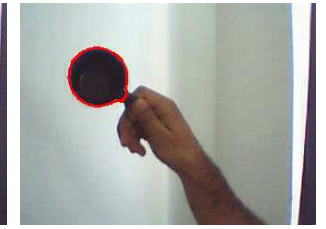

(d) frame 191

Fig. 6. Tracking of a cup.

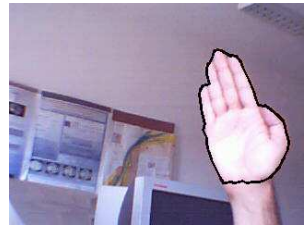

(a) frame 1

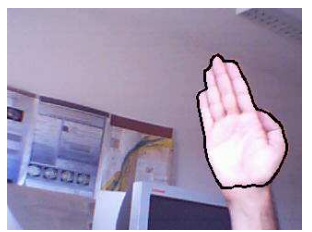

(b) frame 3

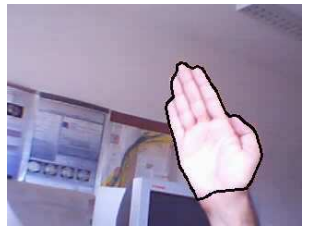

(f) frame 44

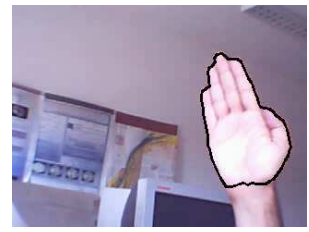

(c) frame 10

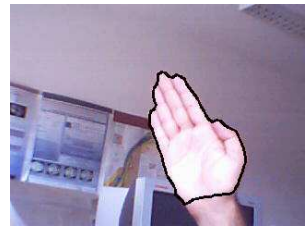

(g) frame 58

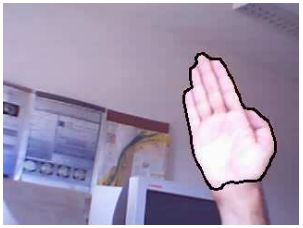

(d) frame 23

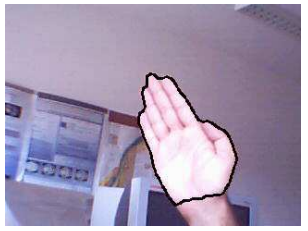

(h) frame 63

Fig. 7. Tracking of a moving hand with fixed camera.

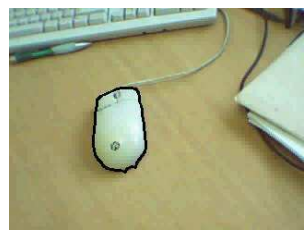

(a) frame 84

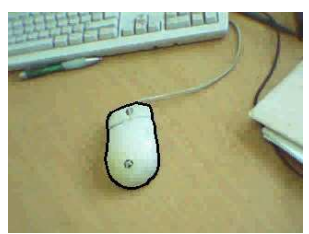

(e) frame 229

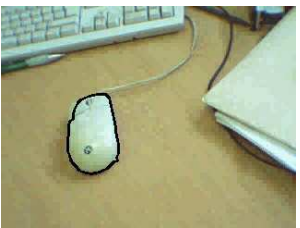

(b) frame 108

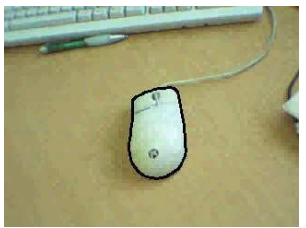

(f) frame 252

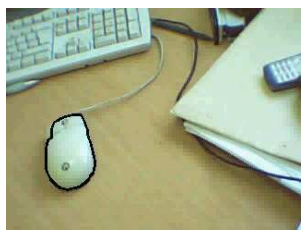

(c) frame 162

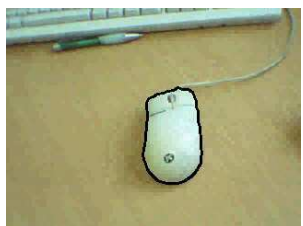

(g) frame 270

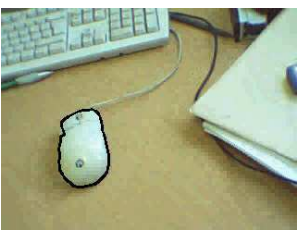

(d) frame 189

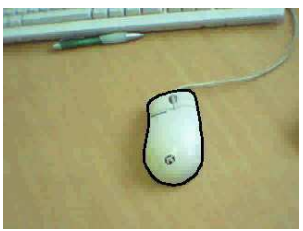

(h) frame 274

Fig. 8. Tracking of a mouse with a moving camera.

This sequence illustrates the model ability to track objects with small shape deformations. Figure 7 shows some frames of a "hand sequence" moving on a relatively complex background. The hand keeps approximatively the same shape in all frames. In the last sequence (Figure 8), we tracked a mouse on a textured background with a moving camera, which illustrates another kind of deformation.

Table 1 summarizes the algorithm parameter values for the three sequences. Except the number of nodes which depends on the shape complexity, all 
Table 1

Parameter values in tracking experiments.

\begin{tabular}{|l|c|c|c|c|}
\hline & nodes & $c_{1}$ & $c_{2}$ & $c_{3}$ \\
\hline Cup sequence & 50 & 0.1 & 0.25 & 1 \\
\hline Hand sequence & 80 & 0.1 & 0.25 & 1 \\
\hline Mouse sequence & 50 & 0.1 & 0.25 & 1 \\
\hline
\end{tabular}

weighting coefficients are equal. This shows that the algorithm is not very sensitive to them. In these applications, we do not use any predication method and all frames are treated with a rate of 25 frames per second. The snakes with shape prior succeeds to pursuit the object when the motion is limited. Larger motion can be handled by using a predictive method such as Kalman filter.

\section{Conclusion and perspectives}

A novel method of snakes with shape prior has been presented in this paper. The originality comes from the embedding of prior knowledge into the model using Fourier based shape descriptors. As demonstrated by our experiments, the proposed method is able to make the snake evolve in concave boundaries and even highly concave boundaries. GVF [27] fails in overcoming highly concave boundaries. In addition, the robustness to noise was enhanced.

However, the used prior does not ensure the invariance to the starting point of the curve which imposes hard constraints on the initialisation (i.e. the initialisation and the template must have the same starting point, a small shift can be corrected). In addition, the complexity of the algorithm is higher compared to classical model of Kass et al. [17]. This is because it requires the re-sampling and computation of the DFT at each iteration. However, time computation remains lower than the GVF snakes. The method is traceable in nearly real-time applications as it was shown for object tracking.

We plan to solve first the dependence to the starting point of the curve. In addition, we intend to extend the model to more general transform such as affine transform, especially the complete family of affine-invariant Fourier descriptors proposed in [3]. Tracking results can be enhanced by the use of prediction methods such as Kalman filter, we could apply the whole method to specific applications such as iris or lips tracking. 


\section{References}

[1] A. Amini, S. Terhani and T. Weymouth, Using dynamic programming for minimizing the energy of active contours in presence of hard constraints, in Proc. 2nd Int. Conference on Computer Vision, (pp. 95-99), Tampa, Florida, 1988.

[2] R. Casselles and R. Kimmel, Geodesic active contours, in Proc. of the Int. Conf. on Computer Vision, (pp. 694-699), Cambridge, Massachusetts, June 1995.

[3] F. Chaker, M.T. Bannour and F. Ghorbel, A complete and stable set of affine-invariant Fourier descriptors, in Proc. of the 12th Int. Conf. on Image Analysis and Processing (ICIAP'03), (pp. 578-581), Barcelona, Spain, 17-19 September 2003.

[4] T. J. Cham and R. Cipolla, Stereo coupled active contours, in Proc. of the IEEE Int. Conf. on Computer Vision and Pattern Recognition, (pp. 1094-1097), San Juan, Puerto Rico, 1997.

[5] L. Cohen, On active contour models and balloons, Graphical Models and Image Processing, vol. 53, no. 2, pp. 211-218, March 1991.

[6] L. Cohen and I. Cohen, Finite-elements methods for active contour models and balloons for 2-D and 3-D images, IEEE trans. on Pattern Analysis and Machine Intelligence, vol. 15, no. 11, pp. 1131-1147, November 1996.

[7] D. Cremers, F. Tischhauser, J. Weickert and C. Schnorr, Diffusion snakes: introducing statistical shape knowledge into the Mumford-Shah functional, Int. J. of Computer Vision, vol. 50, no. 3, pp. 295-313, December 2002.

[8] T. R. CRimmins, A complete set of Fourier descriptors for twodimensional shapes, IEEE trans. on Systems, Man, and Cybernetics, vol. 12, pp. 848-855, 1982.

[9] S. Derrode, M. A. Charmi and F. Ghorbel, Fourier-based invariant shape prior for snakes, in Proc. of the IEEE Int. Conf. on Acoustic, Speech and Signal Processing, Toulouse, France, 14-19 May, 2006.

[10] A. Foulonneau, P. Charbonnier and F. Heitz, Affine-invariant geometric shape priors for region-based active contours, IEEE Trans. on Pattern Analysis and Machine Intelligence, vol. 28, no. 8, pp. 1352-1357, August 2006.

[11] M. Gastaud, M. Barlaud and G. Aubert, Combining shape prior and statistical features for active contour segmentation, IEEE trans. on Circuits and Systems for Video Technology, vol. 14, pp. 726-734, May 2004.

[12] M. Gelautz and D. Markovic, Recognition of object contours from stereo images: an edge combination approach, in 2nd Int. Symp. on 3D Data Processing, Visualization, and Transmission, Thessaloniki, Greece, September 2004.

[13] F. Ghorbel, Stability of invariant Fourier descriptors and its inference in the shape classification, in 11th Int. Conf. in Pattern Recognition, The Hague, The Netherlands, 30 August - 3 September 1992. 
[14] F. GHORBEL, Towards a unitary formulation for invariant image description: application to image coding, Annals of telecommunications, vol. 53, no. 3, pp. 143-153, May 1998.

[15] S.R. Gunn and M.S. Nixon, A dual active contour for head boundary extraction., in Colloq. on Image Processing for Biometric Measurement, London, UK, (pp. 6/1-4), November 1994.

[16] H. JiAng and M.S. DREW, A predictive contour inertia snake model for general video tracking, in Proc. of the IEEE Int. Conf. on Image Processing, Rochester, New York, (pp. 413-416), July 2002.

[17] M. Kass, A. Witkin and D. Terzopoulos, Snakes: active contour models, Int. J. of Computer Vision, vol. 1, no. 4, pp. 321-331, January 1988.

[18] S. H. Kim, J. H. Choi, H. B. Kim and J. W. Jung, A new snake algorithm for object segmentation in stereo images, in Proc. of the IEEE Int. Conf. on Multimedia and Expo, (pp. 27-30), Taipei, Taiwan, June, 2004.

[19] B. Leroy, I. Herlin and L. D. Cohen, Multi-resolution algorithms for active contour models, in Proc. 12th Int. Conf. Analysis and Optimization of Systems, Paris, France, 1996.

[20] N. Paragios and M. Rousson, Shape priors for level set representations, in Proc. of the European Conf. on Computer Vision, (pp. 78-92), Copenhagen, Danemark, 27 May - 2 June 2002.

[21] E. Persoon and K. S. Fu, Shape discrimination using Fourier descriptors, IEEE trans. On Pattern Analysis and Machine Intelligence, vol. 8, n. 3, pp. 388-397, 1986.

[22] N. Peterfreund, Robust tracking of position and velocity with Kalman snakes, IEEE Trans. Pattern Analysis and Machine Intelligence, vol. 21, no. 6, pp. 564-569, June 1999.

[23] N. Ray and S.T. Acton, Active contours for cell tracking, in Proc. Fifth IEEE Southwest Symp. on Image Analysis and Interpretation, Santa Fe, New Mexico, USA, 7-9 April 2002.

[24] M. Rochery, I. JERMYN and J. Zerubia, Higher order active contours, Int. J. of Computer Vision, vol. 69, no. 1, pp. 27-42, August 2006.

[25] L. Staib and J. Duncan, Boundary finding with parametrically deformable models, IEEE trans. on Pattern Analysis and Machine Intelligence, vol. 14, no. 11, pp. 1061-1075, November 1992.

[26] D. J. Williams and M. Shah, A fast algorithm for active contours, Graphical Models and Image Processing, vol. 51, no. 1, pp. 14-26, 1992.

[27] C. Xu and J.L. PRInce, Generalized gradient vector flow external forces for active contours, Signal Processing, vol. 71, n. 2, pp. 131-139, 1998.

[28] C.T. ZAhn and R.Z. Roskies, Fourier descriptors for plane closed curves, Transactions on Computers, vol. 21, n. 3, pp. 269-281, March 1972.

[29] X. Zhong, S. Li and E. TEOH, AI-Eigensnake: an affine-invariant deformable contour model for object matching, Image and Vision Computing, vol. 20, no. 2, pp. 77-84, 2000. 